\title{
Relativity of Energy
}

\author{
Alireza Jamali* \\ Senior Researcher \\ Natural Philosophy Department, Hermite Foundation ${ }^{\dagger}$ \\ alireza.jamali.mp@gmail.com
}

November 29, 2021

\begin{abstract}
After proposing the Principle of Minimum Gravitational Potential, in a pursuit to find the explanation behind the correction to Newton's gravitational potential that accounts for Mercury's orbit, by finding all the higher-order corrections it is shown that the consequences of the existence of speed of light for gravity are not yet fully explored.
\end{abstract}

Keywords - minimum gravitational potential, Mercury's orbit, massenergy space, function space

\section{Contents}

1 Refinement of definition of gravitational potential 2

2 A Physical Principle 3

3 Corrections to Newtonian gravitational potential 4

4 Corrections to Coulomb potential 5

5 Kinematics of Mass-Energy space 5

5.1 The arising metric and its consequences . . . . . . . . 6

5.2 Coordinates ..................... 7

5.3 The transformations . . . . . . . . . . . 7

6 Dynamics in Mass-Energy space 9

6.1 Specific angular momentum . . . . . . . . . . . . 9

${ }^{*}$ Corresponding author

†3rd Floor - Block No. 6 - Akbari Alley - After Dardasht Intersection - Janbazané Sharghi - Tehran - Iran 
7 Consequences for General Relativity

\section{Refinement of definition of gravitational potential}

The theory that I develop in this paper can be approached from several perspectives, but I chose the most 'natural' route that is fair to historical evolution of ideas.

In a previous paper[1] I proposed the idea of heat being a fundamental field sourced by entropy. The merits of that theory indicate that

$$
S \equiv m, \quad T \equiv \phi,
$$

where $S$ is the entropy of a particle, $m$ mass of a particle, $T$ temperature scalar field and $\phi$ gravitational potential, is a true harmony of nature's phenomena. As such it holds both ways: $T \equiv \phi$ implies that all the machinery of statistical mechanics[2] is at our disposal and applicable to mass and gravity. In particular

$$
m=m_{P} \log W
$$

where $m_{P}$ is the Planck mass; and

$$
\phi=\frac{d V}{d m}
$$

where $\phi$ is the (Newtonian) gravitational potential, $V$ is the gravitational potential energy, and $m$ mass of the particle ${ }^{1}$ under the influence of gravitational field $\phi$. Note that (1) is perfectly compatible with the classical definition, as it coincides with

$$
\phi=\frac{V}{m}
$$

for a linear function

$$
\begin{aligned}
V: \mathbb{R} & \rightarrow \mathbb{R} \\
m & \mapsto V(m) .
\end{aligned}
$$

${ }^{1}$ Maybe 'particles' is a better word, as we are now in fact dealing with a continuum of masses. 


\section{A Physical Principle}

Principle. Minimum Gravitational Potential ${ }^{2}$

In flat spacetime, Newtonian gravitational potential ${ }^{3} \phi$ has the absolute minimum $-c^{2} / 2$.

Mathematical implementation of the Principle of Minimum Gravitational Potential leads us to

Definition. Phi factor

$$
\Phi=\frac{1}{\sqrt{1+\frac{2 \phi}{c^{2}}}}
$$

A question might now arise 'why extremum principles must be applied via this particular function?' There are two reasons that single out $\sqrt{1-u(x)}$ :

- Induction: it has worked once with special relativity, therefore the probability that it works again is sure to be greater than zero.

- To implement these kind of principles, we need a function with a bounded domain. There are two functions among all 'elementary functions' of mathematics that have a bounded domain: Logarithm and Square root. Logarithm does not allow the domain to be compact, i.e. for the variables to reach the extremum itself.

Consequently $\phi$ becomes a bounded function $\phi: \mathbb{R}^{4} \rightarrow \mathbb{R}$

$$
\forall \mathbf{x} \in \mathbb{R}^{4}, \quad-\frac{c^{2}}{2} \leq \phi(\mathbf{x}) \leq 0
$$

This $\Phi$ factor is not entirely new: it reminds one of

$$
g_{00}=1+\frac{2 \phi}{c^{2}}
$$

in the weak-field (Newtonian) limit of general relativity[3]. The pesky numerical 2 factor difference between general relativity and Newtonian gravitation continues to trouble us, as usual: if we are to get the new term that arises from the correction[4]

$$
V=-\frac{G M m}{r}\left(1+\frac{3 G M}{c^{2} r}\right),
$$

${ }^{2}$ This principle is in fact a sub-principle of the Principle of Ontologic Finiteness which will be stated in another paper, and justified to follow from the Epistemological Principle of Finiteness, which in turn can be considered a consequence of Descartes' notion of res extensa and Kant's a priori intuitions.

${ }^{3}$ Technically it should be potential difference as only differences in potentials have physical significance, but here we have implicitly assumed the origin to be at $\infty$, viz.

$$
\phi(\mathbf{x})=\frac{V}{m}=\frac{1}{m} \int_{\infty}^{r} \mathbf{F} \cdot d \mathbf{x}=\frac{1}{m} \int_{\infty}^{r} \frac{G m M}{x^{2}} d x=-\frac{G M}{r}=-\frac{G M}{\|\mathbf{x}\|} .
$$


which accounts for the precession of the perihelion of Mercury without any need to consider general relativity ${ }^{4,5}$, we must take a slightly different $\Phi$ factor

$$
\Phi=\frac{1}{\sqrt{1+\frac{4 \phi}{c^{2}}}}
$$

But to make connection with the metric of general relativity via

$$
g_{00}=1+\frac{2 \phi}{c^{2}}
$$

we have to choose the numerical factor to be 2 . A decisive answer cannot yet be given to the question of the true numerical factor but as

$$
-\frac{c^{2}}{2} \leq-\frac{c^{2}}{4}
$$

we choose $-c^{2} / 2$ to allow for a wider range.

\section{Corrections to Newtonian gravitational potential}

As a consequence of the $\Phi$ factor, gravitational potential energy of a particle in a gravitational field $\phi$ is given by

$$
\begin{gathered}
V=-\frac{m c^{2}}{2}\left(\frac{1}{\sqrt{1+\frac{4 \phi}{c^{2}}}}-1\right) \\
=m \phi-\frac{3}{c^{2}} m \phi^{2}+\frac{10}{c^{4}} m \phi^{3}+\mathcal{O}\left(\phi^{4}\right)
\end{gathered}
$$

Expression (4) is well justified as it provides a firm physical ground for (3). To see this, in (4), let

$$
\phi=-\frac{G M}{r}
$$

thus

$$
V=-\frac{m c^{2}}{2}\left(\frac{1}{\sqrt{1+\frac{4 \phi}{c^{2}}}}-1\right) \approx-m \frac{G M}{r}-\frac{3}{c^{2}} m\left(\frac{G M}{r}\right)^{2}-\frac{10}{c^{4}} m\left(\frac{G M}{r}\right)^{3},
$$

up to terms third order in $\phi$.

So far there has been no physical explanation as to why this new term assumes this particular form, especially coefficient 3, nor the next term

\footnotetext{
${ }^{4}$ Of course it is plausible to suppose that general relativity must have somehow assimilated this principle; in a way which is still unknown to me.

${ }^{5}$ This is not to claim that we have 'an alternative' for general relativity -a label that is used like 'communist' to mark heresy-. Light deflection for example, cannot be explained by this modification of gravitational potential. We are simply trying to find a root for this correction, guided by the methodological principle that nothing in theoretical physics works by chance.
} 
could have been found; the new term in (3) is found via comparison with the result of general relativity. I do not shy away from admitting that the number 4 in (4) is still not possible to be derived from anything other than comparison with the result of general relativity ${ }^{6}$, but it is easy to see that the form of the Principle of Minimum Gravitational Potential is unaltered whether one is aware of general relativity or not: there is a unique fundamental constant of nature that has the same dimensions with the gravitational potential (energy per mass), therefore someone in 1900 s can arrive at this principle up to a numerical factor.

\section{Corrections to Coulomb potential}

Principle. Maximum Coulomb Potential In flat spacetime Coulomb potential has the absolute maximum $c^{2} /\left(2 \sqrt{4 \pi \epsilon_{0} G}\right)$, viz.

$$
\forall \mathbf{x} \in \mathbb{R}^{4}, \quad 0 \leq \varphi(\mathbf{x}) \leq \frac{c^{2}}{2 \sqrt{4 \pi \epsilon_{0} G}}=: \frac{c^{2} \sigma_{0}}{2}=\frac{V_{P}}{2},
$$

where $V_{P}$ is the Planck voltage.

Consequently a particle in a Coulomb potential (static electric field) has the following potential energy (voltage)

$$
V=\frac{\sigma_{0} q c^{2}}{2}\left(\frac{1}{\sqrt{1-\frac{4 \phi}{\sigma_{0} c^{2}}}}-1\right)
$$

Which can be considered as yet another testament for my addition of $q$ to Newton Second Law[5]

$$
\mathbf{F}=\left(m+\sigma_{0} q\right) \mathbf{a},
$$

for (5) implies that for a free (not under influence of an electric field) charged particle we have

$$
E=q \sigma_{0} c^{2}
$$

\section{Kinematics of Mass-Energy space}

From (special) relativity we know that once you have the gamma factor

$$
\gamma(\dot{\mathbf{x}})=\frac{1}{\sqrt{1-\frac{\dot{\mathbf{x}}^{2}}{c^{2}}}},
$$

which is the analouge of (2) for us, you can get the metric (and vice versa: you can get the gamma factor from Minkowski metric).

\footnotetext{
${ }^{6}$ Ideally, from an extreme rationalist point of view, the numerical factor too must be derivable from purely theoretical considerations. I have not however yet been able to do this.
} 


\subsection{The arising metric and its consequences}

Using (1), the $\Phi$ factor (2) leads us to the following metric for the massenergy space

$$
d \epsilon^{2}=c^{4} d m^{2}+2 c^{2} d V d m
$$

Accordingly

$$
\left(\frac{1}{c^{2}} \frac{d \epsilon}{d m}\right)^{2}=1+\frac{2}{c^{2}} \frac{d V}{d m}
$$

using (1)

$$
\frac{1}{c^{2}} \frac{d \epsilon}{d m}=\sqrt{1+\frac{2 \phi}{c^{2}}}
$$

We must not forget the transformative nature and consequences of the principle of minimum gravitational potential. The principle is of the same spirit as the second principle of special relativity (constancy of velocity of light for all inertial frames): All 'inertial' observers must agree on a certain minimum for the gravitational potential, we must not forget however that we are considering a different space than spacetime, hence the very notion of 'inertial' changes and must be defined in this space.

To see that we are dealing with certain transformations, divide both sides of (4) by $m$, note that from the old definition $\phi=V / m$, so (4) is really giving us another $\phi$. We have

$$
\tilde{\phi}=\frac{c^{2}}{\sqrt{1+\frac{2 \phi}{c^{2}}}} ;
$$

these transformations however, cannot be the result of a Lorentz transformations, for $\phi$ is a scalar function and must not change under Lorentz transformations. There are two possible solutions for this problem:

- $\phi$ and for that matter $V$, is not really a scalar. It is but a component of some vector.

- $\phi$ is a scalar function, but its functional nature points us to consider it in a function space in which it is a 'vector', hence it is no longer required there to remain invariant.

The first solution does not bring any new mathematics and solves the problem in complete analogy to special relativity. The second solution suggests the use of infinite-dimensional manifolds hence requires new mathematics and introduction of a metric on the space in which $\phi$ is a vector. Moreover by comparing the second solution with special relativity and using a 'historical thought experiment' we can see that the second solution is yet to be applied to special relativity itself! Imagine you are somewhere in a history in which you only have one spatial dimension, you do not consider time to be a proper dimension, and have found a transformation

$$
x^{\prime}=\frac{x-v t}{\sqrt{1-\frac{v^{2}}{c^{2}}}},
$$

you are amazed 'why on earth a scalar is being transformed?' Apart from special relativity you can well take the other route: as you are quite used 
to $x(t)$, 'well, maybe I should consider the function space of $x(t) \mathrm{s}$ in which $x(t)$ is not a scalar'. You will be guided by the Pythagorean theorem to introduce the following distance on the function space of $x(t) \mathrm{s}$

$$
d s=\sqrt{\left(x^{\prime}(t)-x(t)\right)^{2}} d t=\left|x^{\prime}(t)-x(t)\right| d t \Rightarrow s=\int\left|x^{\prime}(t)-x(t)\right| d t,
$$

which is quite expected as this is one of the natural distances for a function space[6]. As we see this perspective requires beginning a whole new exciting investigation, and for this reason we will consider it elsewhere. Back to the meaning of 'inertial' in the mass-energy space, by analogy

$$
\phi \sim \mathbf{v} \text { and } m \sim t,
$$

as

$$
\frac{d \mathbf{v}}{d t}=\frac{d^{2} \mathbf{x}}{d t^{2}}=0
$$

defines the notion of inertia in spacetime,

$$
\frac{d \phi}{d m}=\frac{d^{2} V}{d m^{2}}=0
$$

must define 'inertia' as well in the mass-energy space. We pursue this in the next section.

\subsection{Coordinates}

Following the first approach (relativistic methodology) we introduce the following natural coordinates on the mass-energy space

$$
V^{a}=\left(c^{2} m, V\right), \quad a=1,2 .
$$

\subsection{The transformations}

To fine the explicit transformations in the mass-energy space which respect the principle of minimum gravitational potential, note that geometry of (6) is the same as Minkowski geometry, for one can consider Minkowski metric as

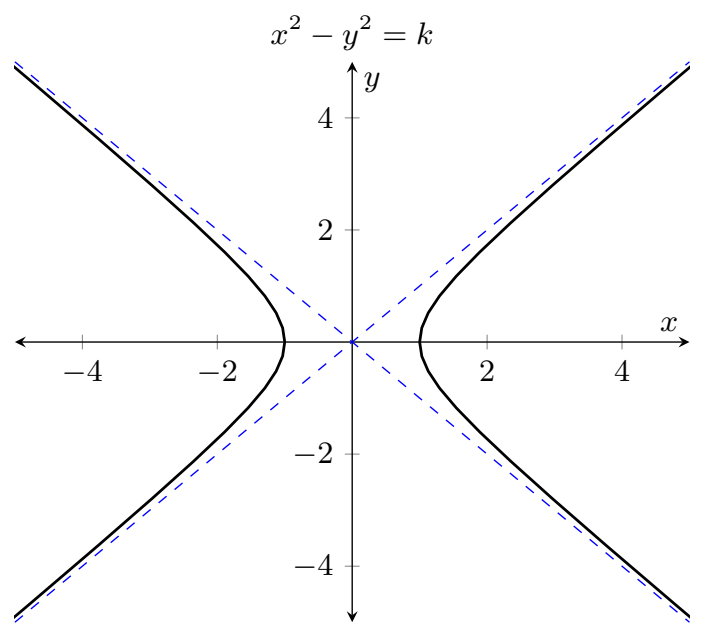


and geometry of (6) as

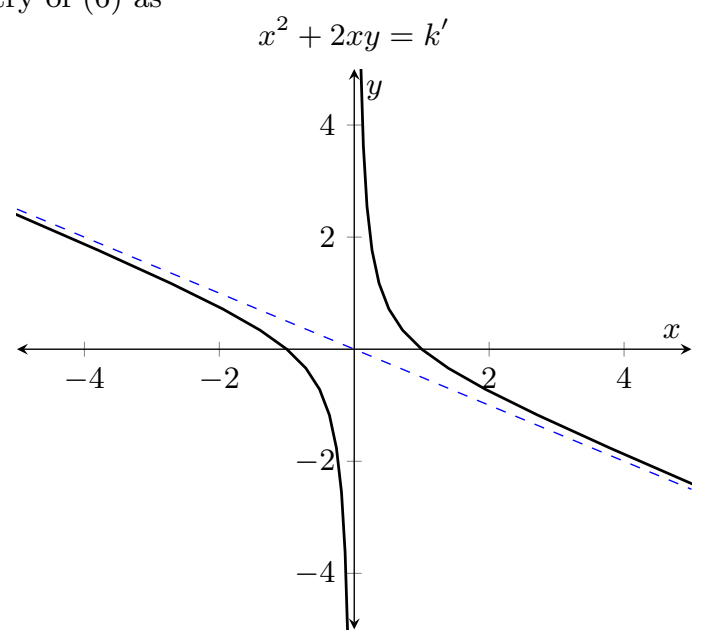

Meaning that (6) is the result of a $\pi / 4$ 'rotation' on Minkowski metric. Accordingly the transformations that leave (6) invariant are linear ones, hence

$$
\left\{\begin{array}{l}
V^{\prime}=A V+B m c^{2} \\
c^{2} m^{\prime}=D V+F m c^{2}
\end{array}\right.
$$

Demanding the transformations to leave (6) invariant means

$$
\left(m c^{2}\right)^{2}+2 c^{2} V m=\left(m^{\prime} c^{2}\right)^{2}+2 c^{2} V^{\prime} m^{\prime},
$$

substituting from (11), we have

$D^{2} V^{2}+F^{2}\left(m c^{2}\right)^{2}+2 F D V m c^{2}+2\left(A V+B m c^{2}\right)\left(D V+F m c^{2}\right)=\left(m c^{2}\right)^{2}+2 c^{2} V m$

$=D^{2} V^{2}+F^{2}\left(m c^{2}\right)^{2}+2 F D V m c^{2}+2 A D V^{2}+2 A F V m c^{2}+2 B D V m c^{2}+2 B F\left(m c^{2}\right)^{2}$

Factorizing $V^{2},\left(m c^{2}\right)^{2}$ and $2 V m c^{2}$ terms and equating coefficients of the same degree,

$V^{2}\left(D^{2}+2 A D\right)+\left(m c^{2}\right)^{2}\left(F^{2}+2 B F\right)+2 c^{2} V m(F D+A F+B D)=\left(m c^{2}\right)^{2}+2 c^{2} V m$

we get

$$
\left\{\begin{array}{l}
D^{2}+2 A D=0 \\
F^{2}+2 B F=1 \\
F D+A F+B D=1
\end{array}\right.
$$

Write the second equation in (13) as

$$
(F+B)^{2}-B^{2}=1
$$

suggesting

$$
B=\sinh \Lambda
$$

using the remaining equations in (13), we have

$$
F=\cosh \Lambda-\sinh \Lambda
$$




$$
A=-\frac{D}{2}=\cosh \Lambda-\sinh \Lambda
$$

Letting $V=0$ in (11) and substitution, leads

$$
\frac{2 \phi}{c^{2}}=\frac{2}{\operatorname{coth} \Lambda-1}=: \alpha
$$

We can now see that the following set of transformations hold between rapidity parameter $\Psi$ of Minkowski geometry and $\Lambda$ of ours:

$$
\left\{\begin{array}{l}
\cosh \Psi=\cosh \Lambda-\sinh \Lambda \\
\sinh \Psi=\sinh \Lambda .
\end{array}\right.
$$

\section{Dynamics in Mass-Energy space}

It is clear by now that potential energies have their own space and geometry, the mass-energy space. To use the analogy (8), first define proper mass from (6)

Definition. Proper Mass

$$
d \mu:=\frac{d \epsilon}{c^{2}}
$$

then

$$
\frac{d \mu}{d m}=\Phi
$$

Definition. Two-potential

$$
\phi^{a}:=\frac{d V^{a}}{d \mu}
$$

\subsection{Specific angular momentum}

Definition. Specific angular momentum

$$
h^{a}:=t \frac{d V^{a}}{d \mu},
$$

where $t$ is time.

From this perspective, conservation of specific angular momentum is easy to see, as

$$
\frac{d h^{a}}{d t}=\frac{d V^{a}}{d \mu}=\text { const. }
$$




\section{Consequences for General Relativity}

Although general relativity is diffeomorphism-invariant under arbitrary coordinate transformations of spacetime, it does not take into consideration the principle of minimum gravitational potential since this principle demands a whole new space, different from spacetime. Be careful not to confuse this with additional dimensions: it is (alway) true that diffeomorphism invariance holds in arbitrary dimensions, but by the virtue of principle of minimum gravitational potential we must talk about diffeomorphism invariance of a whole new manifold.

Recalling that in general relativity

$$
\phi \longrightarrow g_{\mu \nu}
$$

meaning that -as a result of the above discussions- we must also consider diffeomorphism invariance of the manifold of metric tensors. It is in this sense that the principle of general covariance must be demanded: the form of general relativity holds for both spacetime and mass-energy space. We can therefore see in advance that general relativity must be supplemented with another field equation for the mass-energy space; analogue of the Einstein Field Equations.

As an important consequence of the mass-energy manifold (and its diffeomorphism invariance) notice that this new manifold of metrics has its own metric. Accordingly we must deal with two metric tensors if we are to consider mass and energy (mass-energy space) properly alongside general relativity. This is in perfect harmony with my earlier proposal[7] that I argued we need two metrics to solve the problem of energy-momentum of spacetime.

\section{Conclusion and Discussion}

We showed that empirical validity (the correction (3)) goes hand in hand with a theoretical principle (minimum gravitational potential) to lead us to a whole new class of transformations, and -consequently- the notion of mass-energy space which is the most logically-promising approach for the solution of the problem of energy-momentum of spacetime. In this paper we only aimed to show the necessity of this new notion of relativity and some of the essential results, however, the consequences of this proposal are far-reaching and are yet to be explored.

\section{References}

[1] Alireza Jamali. On fundamentality of heat. Preprints.org, 2021.

[2] A.J. Berlinsky and A.B. Harris. Statistical Mechanics. Springer Nature Switzerland AG, 2019.

[3] Ronald J. Adler. General Relativity and Cosmology. Springer Nature Switzerland AG, 2021. 
[4] James D. Wells. When effective theories predict: the inevitability of mercury's anomalous perihelion precession. arXiv:1106.1568 [physics.hist-ph], 2016.

[5] Alireza Jamali. Geometrisation of electromagnetism. viXra:210\%.0132, 2021.

[6] Vladimir Kadets. A Course in Functional Analysis and Measure Theory. Springer International Publishing AG, 2018.

[7] Alireza Jamali. Ontological necessity of nonmetricity: a sketch. viXra:210\%.0166, 2021. 